D. Ganten

\title{
The Journal of Molecular Medicine: tradition, continuity, and renaissance
}

In 1864 L. C. Posner, L. Waldenburg, and C. A. Ewald founded the medical journal Berliner Klinische Wochenschrift. The journal rapidly became one of the leading publications in clinical medicine, one in which many landmark articles were published. In 1922 the Berliner Klinische Wochenschrift merged with the Therapeutische Monatsheft, edited by W. Heubner, L. Langstein, and E. Meyer to become the Klinische Wochenschrift, edited by V. Salle, A. Gottstein, A. von Domaris, P. Jungmann, C. von Noorden, H. Schwiegk and others. The Klinische Wochenschrift was the most important forum for the publication of articles and reports in clinical medicine in the German language. It was one of the few journals in which basic science as well as patient-oriented clinical research was published. For this reason the (Gesellschaft Deutscher Naturforscher und Ärzte (Society of German Natural Scientists and Physicians) adopted the Klinische Wochenschrift as its official journal. Many prominent scientists served as editors and editorial board members in the journal's long productive history. The tradition of maintaining high standards for medical publications, including Nobel prize-winning papers, has thus continued from 1864 to the present day.

In the second half of this century it became clear that English was the lingua latina of the natural sciences, biology, and medical research. Therefore, English was adopted as the language in which the Klinische Wochenschrift was to appear. The title of the journal was changed to the Clinical Investigator by the previous editor, Prof. Nepomuk Zöllner. Professor Zöllner, a prominent clinician and scientist at the Ludwig Maximilian University in Munich, admirably steered the journal from its traditional and national German history to an international publication. A careful balance between basic sciences and clinical medicine was maintained.

The goal of the journal will continue to be the bringing together of basic science and clinical medicine. The focus will be more oriented towards molecular biology

D. Ganten

Max-Delbrück Center for Molecular Medicine,

Robert-Rössle-Strasse 10, D-13122 Berlin, Germany and gene technology, which have become particularly important for the progress of modern medicine in all its aspects. We will assure an orientation towards patient-related research and will continue to encourage contributions from clinicians.

The Journal of Molecular Medicine publishes highquality original papers, rapid communications, review articles, and correspondence relevant to all aspects of human biology and pathophysiology. The application of research involving gene technology, gene therapy, molecular structural analysis, genetic epidemiology, and molecular and clinical pharmacology has made unprecedented progress in the understanding, prevention, diagnosis, and treatment of human diseases. These areas will therefore be given particular attention by the editorial board. Papers describing in vitro or animal studies will be accepted if they are relevant to human physiology and pathology.

To fulfill this task, the Journal of Molecular Medicine is published monthly. The editorial policies and practices of the Journal of Molecular Medicine are established by the publisher (Springer-Verlag), the editor in chief, the associate editors, and the large international editorial board, which includes practicing physicians and clinician-scientists as well as hard-core basic scientists and molecular biologists.

Criteria for manuscript acceptance include originality and interest to the broad readership of the Journal of $\mathrm{Mo}$ lecular Medicine in addition to high quality and scientific rigor. The editors will rely fully on the reviewers to assign priority to the submitted manuscripts and will do their best to judge the manuscripts in the fairest possible manner.

The Journal of Molecular Medicine will publish, without page charges, especially solicited commentaries, editorials, perspectives, and articles reporting original data. Regular articles and rapid communications are handled similarly in the review process; however, rapid communications will be given relative priority and will receive expedited publication after acceptance. This practice will decrease the time required to final publication. 
The review process is critical to the quality of a journal. Requests by authors to exclude certain potential reviewers will be honored if these requests appear reasonable. Further, authors may propose a list of potential expert reviewers. The manuscripts are sent to two or three reviewers, and the results of the review process are discussed by the associate editors in regular meetings. Reviewers are asked to screen the manuscripts immediately and to assign a priority based on quality, originality, and relevance to the readership. Their comments are promptly forwarded to the editorial office by fax transmission. Papers of insufficient quality are rejected after this initial screening process. The screening review has been adopted by several journals and is aimed at providing an expedited review process that is fair and in the best interests of the authors and reviewers. Complete reviews with the decision of the editorial board will be forwarded to the authors in the shortest time possible. Papers may be accepted with no change, or provisionally accepted pending minor revisions.

Manuscripts requiring major revisions are subjected to careful reexamination, and no guarantee is made about their ultimate acceptance. If the authors of a rejected manuscript believe a serious misunderstanding or scientific error has occurred during the review process, a letter of rebuttal may be sent to the editorial office. and the arguments will be reexamined. The review process is handled in a rigorously anonymous way. and the identity of the associate editors and reviewers involved in the decisions is kept strictly confidential. We also ask the authors to keep verbal communication with the editorial office to a minimum to maintain objectivity. This practice will also insure well-documented interactions between the editorial office and the authors.

To make frequent editorial meetings possible. we have decided to ask several scientists in the Berlin area to serve as associate editors. We understand that this move could raise concerns of regionalism or bias. To avoid any possible appearance of favoritism. manuscripts submitted from these research institutions will be handled separately, and the minutes of the editorial meeting will be communicated to the international associate editors, who will closely interact with the Berlin editorial office.

The Journal of Molecular Medicine, its editor, associate editors, and members of the editorial board rigorously observe the principles of scientific integrity. We are convinced that scientific fraud and false publications are rare events: however, if discovered. we will take appropriate action. The scientific peer review process has its inherent flaws and weaknesses; however, external peer review remains a tried and true method of maintaining excellence. The Journal of Molecular Medicine is committed to promoting the quality and fairness of the review process in every way possible, as well as enhancing its speed and efficiency. We welcome suggestions from the readership to improve this process and we hope for your support in making the Journal of Molecular Medicine one of the leading journals in its field.
Biomedical research is currently undergoing revolutionary changes, with consequences that are not yet fully recognized by everyone. I refer to the advent of molecular medicine, a new type of medicine which makes use of molecular biology and gene technology to study the basic principles of health and disease. Work in this field has resulted in the development of new forms of health preservation, new definitions of diseases, diagnostic possibilities, and therapies which involve the genome itself.

From the history of medicine we are familiar with similar paradigms which have completely changed our basic understanding of health and disease. In medieval times Padua was the center of European medicine. It was here that Andreas Vesalius and Giovanni Morgagni developed modern macroscopic anatomy. Their objects of study, namely the dead, had to be transported in secrecy from the marketplace where convicts were killed or from the cemeteries of the poor without consent of the Catholic Church and society to the autopsy theater of the university. In the autopsy rooms of the anatomy departments of old European universities in cities such as Padua, Siena, Bologna, Montpellier, Paris, Heidelberg, and Prague, the origins of our present day knowledge developed. From these investigations we learned about organ structure and function in health and disease. This was the era of the anatomical and morphological paradigm in medicine.

In the nineteenth century Rudolf Virchow, from the Department of Pathology at the Charite Hospital, the medical college of Berlin University, developed the paradigm of cellular pathology. He fostered the idea that life, health, and disease are determined by the smallest units of organs, namely the cells which regulate organ function. His ideas are still valid for today's concepts of pathophysiology and cell biology.

A further important paradigm developed from Virchow's cellular pathology. namely the notions that molecular and chemical reactions are key to the processes of life, and that diseases are in part consequences of disturbances in the chemical equilibrium of cells. Scientists in the last century and first half of this century, such as Walter Flemming such as Justus Liebig, Paul Ehrlich, Marcelin Berthelot, Felix Immanuel Hoppe-Seyler, Otto Warburg, Barbara McClintock, Adolf Butenandt. Linus Pauling and others undoubtedly had a great influence on the development of these ideas. The biochemistry of medicine developed at the end of the last century. The field had a stormy progress in the 1920s and 1930s, but nevertheless facilitated the development and evolution of modern pharmacology between 1950 and 1980 .

A combination of anatomical. morphological, and biochemical oriented thinking still dominates medicine at the present time. These methods undoubtedly have great merits; however, their limits have become more and more apparent. The development of molecular and genetechnological methods represented a quantum leap in the spectrum of diagnostic and therapeutic possibilities. The term "molecular medicine" was coined to designate the use of molecular biology and gene-technological meth- 
ods for medical research, diagnosis, therapy, and prevention.

Molecular medicine defines health and disease as a consequence of information processing in the genetic program. The human genome consists of 22 chromosomes, the autosomes, and the sex-determining chromosomes $\mathrm{X}$ and $\mathrm{Y}$. These chromosomes are built on the basis of three billion nucleotides. They form about one hundred thousand genes which are the basic information molecules determining the structure of proteins and peptides and are responsible for building the cells, organs, and the whole body, and its development and function.

In the framework of the international human genome project, the human genes will be deciphered within the next ten years. There are a few remarkable peculiarities which make molecular medicine so special. 1. The alphabet of the gene is extremely simple; it consists of only four letters (ATCG) representing the bases adenine, thymidine, cytosine, and guanidine. This simple alphabet of inheritance, the genes, is the important basis for unprecedented physical precision, development of rapid methodologies which have resulted in great advances in knowledge in biology and medicine. 2. In many instances, the genes of man and animals are very similar, or even nearly identical, much more than one would expect from the phenotypic appearance of a rat, camel, or human. This similarity makes it possible to develop very simple but highly informative models for research on clinical questions. Some particularly important genes, such as those responsible for the adaptation of the body to stress, have been preserved throughout evolution of the animal kingdom to man over several million years. 3 . Homology of the genes among various animal species and man enables us to perform experiments on function in simple organisms and models and develop new ideas including new therapeutic interventions and drugs. The "humanization" of transgenic animals is such an example and a change in paradigm in clinical pharmacology. 4 . With respect to therapy, it is possible to leave the traditional application of a chemical substance, such as a drug, and instead transplant molecules, which carry information, thereby enabling the body to synthesize its own therapeutic substance. We term this strategy "gene therapy." The transplantation of information molecules in gene therapy is thus a completely new principle of therapy. The idea is of great promise but is not yet a wonder weapon against cancer and other incurable diseases.

Medical research and clinical medicine are already using the new methods of molecular medicine in their research and daily practice. It is not uncommon for biologists, molecular biologists, and physicians to work together at one bench or to discuss common problems at the bedside. However, one often notices that the basic scientists and clinicians speak two different languages; one does not necessarily understand the other. The Klinische Wochenschrift participated in these developments throughout its history. We hope that its continua- tion, the Journal of Molecular Medicine will continue in this role.

The Springer-Verlag was founded in 1842 by Julius Springer and is one of the oldest publishing houses, with a very proud tradition of sponsoring journals in medicine and other related areas for over 150 years. The Journal of Molecular Medicine, from its inception as Berliner Klinische Wochenschrift from 1884, has undergone many changes in title and content. It saw the changes in the medical paradigms from macroscopic anatomy to cellular pathology and medical biochemistry to molecular biology and finally to molecular medicine.

German reunification has made it possible to resume the long scientific tradition in Berlin-Buch. The Max Delbrück Center for Molecular Medicine (MDC), founded in January 1992 as a national research center, was developed from three institutes of the former East German Academy of Sciences, and is named after the GermanAmerican Nobel prize winner Max Delbrück. Together with the Russian geneticist Nikolai Vladimirovich Timoféeff-Ressovsky, who worked in the Kaiser Wilhelm Institute in Berlin-Buch, Delbrück set out to verify fundamental principles of molecular genetics and published a ground-breaking paper on the nature of gene mutation and gene structure. An old journal thus comes full circle back to Berlin.

With the institution of a new editorial team there are the inevitable changes that reflect the individual touch of the group assuming journal management. Nevertheless, as we begin our term in the 130th year of the journal's existence, we pledge to maintain the excellence and high standards that have been passed down to us from the prominent editors throughout its proud history, especially those of Nepomuk Zöllner, the previous editor from Munich.

We are very enthusiastic about the prospects for developing the Journal of Molecular Medicine as a forum for the exchange of new knowledge. Along with Springer-Verlag and the Gesellschaft Deutscher Naturforscher und Ärzte, each of us feels a tremendous sense of excitement and anticipation of the great things to come in the immediate future for molecular medicine and for our journal.

\section{References}

1. Danks DM (1993) The impact of molecular genetics on clinical medicine. Med J Aust 158:147-148

2. Götze H (1992) Der Springer Verlag Stationen seiner Geschichte Teil II, 1945-1992. Springer, Berlin Heidelberg New York

3. Hiatt H, Goldman L (1994) Making medicine more scientific. Nature 371:100

4. Sarkowski H (1992) Der Springer Verlag Stationen seiner Geschichte Teil I, 1842-1945. Springer, Berlin Heidelberg New York

5. Varki AP (1993) The editorial policies and clinical practices of the Journal of Clinical Investigation. J Clin Invest 92:v-vii 